\title{
Défis scientifiques et sociaux de l'anthropologie maritime
}

Nastassia Reyes, Clément Garineaud et Frédérique Chlous

\section{OpenEdition}

1 Journals

Édition électronique

URL : http://journals.openedition.org/ethnoecologie/3485

DOI : $10.4000 /$ ethnoecologie.3485

ISSN : 2267-2419

Éditeur

Laboratoire Eco-anthropologie et Ethnobiologie

Référence électronique

Nastassia Reyes, Clément Garineaud et Frédérique Chlous, « Défis scientifiques et sociaux de l'anthropologie maritime », Revue d'ethnoécologie [En ligne], 13 | 2018, mis en ligne le 25 juin 2018, consulté le 01 mai 2019. URL : http://journals.openedition.org/ethnoecologie/3485 ; DOI : 10.4000/ ethnoecologie.3485

Ce document a été généré automatiquement le 1 mai 2019.

Revue d'ethnoécologie est mis à disposition selon les termes de la licence Creative Commons Attribution - Pas d'Utilisation Commerciale - Pas de Modification 4.0 International. 


\title{
Défis scientifiques et sociaux de l'anthropologie maritime
}

\author{
Nastassia Reyes, Clément Garineaud et Frédérique Chlous
}

1 Dans le débat public, les enjeux sur l'environnement se font plus présents et le milieu maritime n'échappe pas à cet emballement. Les problématiques liées aux pollutions marines, aux espèces menacées, à la construction des territoires, à l'augmentation des usages de loisirs, aux politiques de conservation, aux droits d'accès aux ressources et aux biens communs, etc. tiennent une place de plus en plus centrale dans les débats sociétaux et sur les agendas politiques. Plus largement, ces questions relèvent des rapports Hommes-Natures, de la définition des pratiques durables et du devenir de l'Homme sur Terre. Dans le champ scientifique, et plus spécifiquement de l'anthropologie, des recherches se développent autour de ces problématiques. Elles prennent en compte les dynamiques sociales et les transformations au sein des sociétés locales, mais aussi les articulations entre les différentes échelles.

2 Les flux, les interactions entre la mer et la terre sont constants. Aussi, analyser les enjeux sur le milieu marin oblige à inscrire une anthropologie maritime dans un cadre plus large et en interaction constante avec les cadres conceptuels de l'anthropologie de la nature, de l'anthropologie politique, de l'anthropologie des techniques, d'une approche phénoménologique tout en intégrant la terre, le climat et les milieux d'eau douce. Qu'en est-il donc de ces autres espaces aquatiques qui échappent à cette conceptualisation du domaine maritime? Se pose explicitement la question de savoir s'il est approprié ou adéquat de renommer ce champ et de s'aventurer à parler d'une anthropologie des mondes aquatiques. Cette désignation serait-elle pertinente ? Ce qui est certain, c'est que les enjeux et les frictions qui viennent à concerner ces milieux se font grandissants et que les travaux qui aujourd'hui participent de l'anthropologie maritime investissent de nouvelles approches et questionnements susceptibles de rediscuter de la portée scientifique de ces recherches. En parallèle, force est de constater que ces enjeux environnementaux et leur importance dans le débat public amènent aussi à se demander s'il est nécessaire de revendiquer l'anthropologie maritime d'un point de vue scientifique ou d'un point de vue sociétal? Il convient de se demander si l'anthropologie maritime 
doit se constituer en tant que champ scientifique circonscrit, en interaction nécessaire avec d'autres et/ou s'il est nécessaire qu'elle intervienne et se fasse entendre dans les débats sociétaux?

3 En guise de conclusion à ce dossier, nous reviendrons tout d'abord sur le contexte d'émergence du colloque, puis nous discuterons des débats scientifiques autour de la délimitation, de la spécificité et de la portée du champ de l'anthropologie maritime. Enfin, nous proposerons de revenir sur la question d'une plus grande visibilité de l'anthropologie maritime dans les débats sociétaux.

\section{Retour sur les prémices du colloque}

4 En 2014, un petit groupe de chercheurs du Muséum national d'Histoire naturelle (MNHN) se réunissent afin d'échanger sur ce qu'est, l'anthropologie maritime. Les chercheurs qui composent ce groupe s'intéressent aux sociétés interagissant et côtoyant des espaces marins et aquatiques. Si l'intérêt pour les pratiques et les représentations liées à la pêche, la collecte d'algues ou de coquillages pouvait constituer un dénominateur commun, les discussions se référaient à plusieurs milieux: la mer, les littoraux, les fleuves ou les lagunes. D'emblée, l'appellation "anthropologie maritime » a posé question et a paru réductrice. À cette diversité, sont venues s'ajouter les approches théoriques particulières de chaque chercheur: certains se réclamant plutôt d'une ethnoécologie, d'autres d'une anthropologie de la nature ou encore d'une anthropologie de l'environnement, mais aussi de la sociologie des sciences... pour n'en donner qu'un aperçu. Les échanges ont alors permis d'identifier que des postures et des intérêts scientifiques pluriels venaient à composer les recherches portant sur ces milieux marins et aquatiques. Face à ce constat, l'organisation d'un colloque devait permettre de préciser les travaux qui composent ce champ et d'étendre la réflexion en impliquant une plus large communauté scientifique.

5 La volonté de définir le champ de l'anthropologie maritime, de construire une réflexion épistémologique, s'explique en grande partie par l'histoire de ce champ au sein du Muséum national d'Histoire naturelle. Le mot d'ouverture de Serge Bahuchet lors du colloque rappelait comment le Centre d'Ethno-technologie en milieux aquatiques (CETMA) constitué autour de Théodore Monod, Jean Abel Gruvel et Paul Budker s'était trouvé adossé à une équipe d'anthropologie maritime dans laquelle Aliette Geistdoerfer ou Jacques Ivanoff, comme d'autres, étaient impliqués. En quoi l'anthropologie maritime avait changé (ou non) en référence à nos illustres devanciers? Était-ce une question de cadrage théorique, de méthodologies mises en œuvre, de questionnements, de terrains fréquentés ou de milieux? L'anthropologie maritime semble dépasser les seuls enjeux strictement marins. L'introduction développée par Hélène Artaud montre en effet la labilité de cette appellation ainsi que les débats conceptuels et les postures des principaux auteurs ayant produit des définitions de ce champ. Malgré les efforts d'Yvan Breton, de James M. Acheson, comme d'autres auteurs, force est de constater que l'analyse des pratiques de pêche reste, en France, l'un des sujets les plus couramment abordés dans le champ de l'anthropologie maritime. Les glissements conceptuels sont parfois tels que «l'anthropologie maritime » vient souvent se confondre avec l'anthropologie des pêches. 


\section{L'anthropologie maritime, un champ disciplinaire en cours de redéfinition}

6 Nous reviendrons ici sur trois des principaux aspects qui transparaissent dans les textes publiés dans ce numéro et qui ont animé les discussions lors du colloque. C'est tout d'abord la spécificité du champ et des objets étudiés par l'anthropologie maritime qui sera discutée, ensuite la pertinence et la dialectique du rapport Terre-Mer fera l'objet d'une analyse et enfin, un troisième aspect concomitant aux deux autres: celui de la délimitation scientifique que pose ce champ de recherche.

\section{L'anthropologie maritime, un champ porteur de spécificités ?}

7 Puisque la question du milieu marin a été centrale dans la construction de l'anthropologie maritime, considérons-la sans délai. Durant ces deux jours, plusieurs qualificatifs ont caractérisé le milieu maritime : espace mouvant, aléatoire, invisible, peuplé d'espèces aux statuts différents, lieu de pratiques et de représentations, objet de politiques publiques et, pourtant, pouvant paraître à certains inoccupé... Mais, qu'en est-il du point de vue des productions scientifiques? La perspective d'un espace inhabité ou inapproprié peut d'emblée être évacuée. Les travaux en sciences humaines s'attachent justement à décrire les interactions humaines en lien avec cet espace et à considérer qu'il s'agit d'un espace social organisé, nommé, porteur de pratiques et de représentations. Ils montrent aussi que l'invisibilité est plus prégnante pour les étrangers au milieu que pour le marin, le nomade de la mer ou le capitaine de marine marchande. En effet, ceux-ci connaissent en trois dimensions l'espace qu'ils pratiquent, grâce aux couleurs particulières, aux profondeurs, aux fonds marins ou par l'intermédiaire des engins de pêche ou de la technologie embarquée.

8 Ce qui a toutefois réuni les échanges durant le colloque tient à la dimension changeante de ce milieu. Cette dimension, peut-elle comme pour les milieux terrestres et les pratiques agricoles constituer l'une des principales spécificités du milieu maritime? D'autres espaces aquatiques détiennent-ils cette même particularité? Les nombreuses recherches montrent comment les pratiques, les techniques et les savoirs des pêcheurs leur permettent d'être dans un rapport particulier à ce milieu. Si l'adaptabilité et l'opportunisme des pêcheurs semblent les singulariser, la régularité et l'irrégularité des marées donnent souvent une spécificité singulière à la dimension maritime. En cela, la mer déroge-t-elle aux autres milieux aquatiques? Mais de là à constituer, autour de cette singularité du milieu, un champ à part entière, n'est-ce pas un peu audacieux ? Cette question témoigne elle aussi de la confusion qui concerne ce champ de recherche : celle d'une adhésion entière des anthropologues à leur objet de recherche. Venons-en alors à l'idée de réinterroger la Mer comme catégorie analytique et de la mettre en regard de son pendant : la Terre.

\section{Rapport Terre-Mer, une dialectique à déconstruire}

Le champ de l'anthropologie maritime s'est appuyé sur des considérations aussi générales que la localisation géographique et les caractéristiques physiques. Alors, la mer est 
mobilisée, en tant que catégorie analytique. Or, cette catégorie s'appuie sur une dualité consubstantielle : celle d'une distinction entre d'une part la Terre et d'autre part, la Mer.

Une telle distinction pose question et l'on peut aussi être circonspect quant à l'idée de penser, d'une part la mer dans une dimension monolithique et d'autre part, comme totalement autarcique. Il existe en effet de nombreuses manières de nommer ce milieu, en fonction de sa distance à la terre, littoral ou hauturier, et les acteurs qui tentent d'investir ce milieu s'inscrivent dans des référentiels cognitifs tout aussi variés. Les travaux en anthropologie ont en effet montré la richesse et la variabilité, dans le temps et selon les lieux, des perceptions qu'ont les populations de ce milieu. Une grande diversité de sociétés interagit avec la mer et des singularités se dessinent en tous lieux. Il en est d'ailleurs de même, si l'on s'intéresse aux études produites sur les espaces terrestres.

11 Par ailleurs, l'article proposé par A. Levain et P. Laval montre que les influences de la mer atteignent pour partie aussi l'intérieur des terres. La mer ne reste pas cantonnée à sa dimension littorale et au large. Elle influe sur la circulation des fleuves, les deltas, ainsi que les lagunes, dépassant la seule limite spatiale du maritime. Les travaux en anthropologie ont démontré que cette dialectique terre-mer n'est pas universelle et qu'elle prend appui sur une représentation dichotomique des espaces qui est profondément occidentalo-centrée. Si la singularité et l'universalité des pratiques et les problématiques existent par-delà les localités, il convient de revoir les cadres analytiques et de dépasser la dialectique terre-mer, ainsi que la catégorie mer. Cette perspective permet d'analyser les rapports des sociétés aux mondes aquatiques comme inscrit dans un continuum et se saisir d'une approche plus intégrée.

\section{Délimitation du champ et prise en compte des enjeux globaux}

12 Compte tenu des limites que pose la dénomination anthropologie maritime, la question de la redéfinition des contours de ce champ est revenue à de multiples reprises. La question de ce qu'est, et ce que n'est pas, l'anthropologie maritime aujourd'hui a été posée. Tandis que d'autres ont tenté de lancer le débat en s'interrogeant, dans une perspective programmatique, sur ce que l'anthropologie maritime pourrait être amenée à devenir.

Lors de l'appel à communication, quatre axes de réflexions ont été proposés. Le premier proposait de s'intéresser aux formes de socialisation et d'appropriation de l'espace maritime ; le deuxième visait à analyser la question du genre dans les activités maritimes, mais aussi dans la détermination sexuée de la mer en tant qu'élément; le troisième proposait de débattre des fondements conceptuels et méthodologiques autour de la redéfinition de ce domaine de recherche et enfin, le quatrième interrogeait la mer comme le lieu d'enjeux et de questionnements globalisés. Ce recensement ne prétendait pas couvrir l'ensemble des sujets relatifs au domaine maritime. Mais toujours est-il que les propositions de communications qui ont été faites se sont surtout reportées au premier et au quatrième axe. Dans ce cadre, les communications autour des sociétés de pêcheurs ont été dominantes. En revanche, les entrées - pourtant classiques - autour de la technologie des pêches ou celle de la parenté n'ont pas trouvé d'échos particuliers.

Certaines thématiques comme celle des flux migratoires par les circuits maritimes, des enjeux pétroliers ou des problèmes de pollution sont restées assez peu investies. Les questions d'ordre géopolitique autour de la piraterie ou des circuits de commercialisations des produits de la mer, n'ont également pas été abordées. Si depuis ces dernières années, le domaine maritime fait l'objet de nouveaux usages, d'un 
élargissement vers la haute mer, et voit l'implication de nouveaux acteurs, les projets en anthropologie qui s'engagent sur ces questions laissent de nombreux sujets encore peu explorés. Il est également possible de se demander si les anthropologues qui étudient ces questions se revendiquent de l'anthropologie maritime?

Force est de constater qu'une anthropologie qui intègre les milieux aquatiques peut apporter, en termes de descriptions et d'analyses de pratiques, d'organisation des territoires et de représentations à la discipline anthropologique dans sa globalité. Les espaces aquatiques présentent des formes d'altérités, ne serait-ce par le milieu, mais aussi les objets de recherche que ces travaux abordent. Les différences liées aux variabilités annuelles, saisonnières ou journalières, avec les milieux terrestres sont intéressantes à mettre en perspective. Une appellation plus intégrative de ce champ permettrait de dépasser les limites conceptuelles que le champ de l'anthropologie maritime pose actuellement, qu'il s'agisse de théorie ou de méthodologie. Pour l'heure, la distinction entre anthropologie maritime et anthropologie des mondes aquatiques a contribué à renouveler la portée heuristique de ce champ. Au-delà des milieux concernés, ce domaine de recherche est en pleine évolution à mesure que se dessinent, depuis ces dernières années, des enjeux sociétaux forts. Les champs de l'anthropologie politique, de la nature, des techniques, de la globalisation et bien d'autres sont mobilisés.

\section{Le maritime au cœur des enjeux de société. Quelle place pour l'anthropologie?}

Dès lors que le regard et les activités humaines se sont tournés vers la mer et les milieux aquatiques, les mondes politiques régionaux, nationaux ou internationaux et scientifiques se sont intéressés à ces espaces. De nouveaux acteurs, considérés comme ayant des compétences particulières dans la recherche, dans les sphères administratives ou dans la représentation de la société civile se sont impliqués dans la connaissance et l'analyse des enjeux maritimes. Les pêcheurs, acteurs séculaires de ces espaces se retrouvent à devoir partager un milieu sur lequel ils avaient développé des connaissances et qu'ils gouvernaient localement. Ces changements participent d'un nouvel ordre social, qui articule différentes échelles autour des espaces maritimes et aquatiques.

Cette dernière partie invite à interroger la nécessité de prise de position dans les débats publics par l'anthropologie maritime et des mondes aquatiques. Nous évoquerons, tout d'abord, le contexte au sein duquel la gouvernance des espaces maritimes mobilise les sciences humaines et sociales. Nous reviendrons ensuite sur la dimension institutionnelle liée à la catégorie "mer». Enfin, nous clarifierons notre posture quant aux cadres qui pourraient permettre à l'anthropologie d'intervenir dans les débats sociétaux.

\section{La mer, une catégorie pourtant opérante dans le domaine institutionnel}

Précédemment, nous avons déconstruit l'opposition terre-mer en montrant qu'elle était peu heuristique et scientifiquement restrictive. En revanche, en dehors des cadres conceptuels scientifiques, la catégorie "mer» s'avère opérante. Celle-ci est en effet continuellement mobilisée au sein des sphères institutionnelles. S'impliquer dans les 
débats publics suppose donc de s'intéresser à cette catégorie jusqu'à porter une réflexion sur son contenu et ses limites.

\section{La gouvernance des espaces aquatiques, revirement vers les considérations humaines}

De nombreux travaux de recherche ont démontré que des systèmes de normes construisent la protection et le partage de la mer. Modernité, progrès, productivité, conservation, mise en valeur, protection de la nature sont les objets de nombreuses politiques nationales et internationales, ne prenant pas toujours en compte la vulnérabilité des populations littorales. Dès lors les politiques, les projets de gouvernance et certaines activités nouvelles entraînent un changement pour les sociétés concernées. Mais, alors qu'elle était souvent délaissée, la dimension socio-économique relative à ces espaces commence à être plus sérieusement considérée au niveau institutionnel. Les approches strictement écologiques de ces milieux ont montré des limites. Dans ce contexte, l'expertise apportée par les sciences humaines et sociales est en passe d'être davantage sollicitée. Cette imbrication de la discipline dans le champ politique pourrait témoigner d'un tournant institutionnel à grande échelle.

Pour l'heure, la recherche en anthropologie est restée relativement en marge de ces dynamiques institutionnelles. Les écologues, comme les halieutes et plus généralement les biologistes marins ont pallié la nécessité d'obtenir des données sur les problématiques sociales en investissant parfois eux-mêmes les méthodes des sciences humaines et sociales. Cela révèle-t-il que la demande d'information sur les problématiques sociales est si importante, que si elle n'est pas investie par les sciences humaines et sociales, elle se voit investie par d'autres? L'anthropologie n'est pas nécessairement invitée dans les débats, pour autant doit-on la laisser en marge? La question corrélative que nous soulevons ici est celle de savoir si nous jugeons nécessaire de faire en sorte qu'elle prenne part aux débats publics ou si au contraire la recherche sur la mer doit rester indépendante de ces enjeux afin de nourrir une réflexion scientifique autonome.

21 Cette réflexion ne doit pas être évacuée tant il est vrai que la demande institutionnelle sur les dynamiques sociales se fait grandissante, et qu'il est certain que d'autres acteurs décideront d'intervenir sur ces aspects. Si l'implication de l'anthropologie dans ces enjeux de gouvernance de l'espace maritime requiert une posture réflexive, son absence mérite aussi une certaine attention tant elle peut être porteuse de sens. En effet, une mise à distance de ces enjeux se résumerait à laisser à d'autres acteurs la possibilité d'intervenir seuls sur un domaine où pourtant, l'anthropologie peut apporter son expertise. Les analyses produites dans le domaine participent à comprendre le monde social et ses évolutions et les rendre intelligibles est primordial. Nous partageons la volonté de créer des synergies et de mutualiser les apports de diverses expériences, autour des enjeux sur les mondes marins et aquatiques.

\section{Pour une science en prise avec les enjeux sociétaux}

On ne peut que rester prudent quant à la dimension performative que peut générer un tel positionnement, toutefois il est évident que cette réflexion est en prise avec les enjeux sociétaux, mais aussi scientifiques actuels. L'objet de cette attention n'est pas de concevoir un repli disciplinaire, tant il est vrai que les réflexions sur le maritime n'ont eu 
de cesse de se nourrir des productions en histoire, en droit ou en économie, mais, simplement de structurer notre positionnement de manière à éclaircir la posture de chercheur que nous souhaitons adopter. Dans les contextes où science et expertise se rejoignent, le chercheur doit veiller à se départir des attentes parfois fortes de certains acteurs de la gouvernance. Celui-ci est parfois sommé d'adopter une posture normaliste qui, du point de vue scientifique, reviendrait à l'amputer de l'un de ses principaux apports : sa capacité de décentrement. Pour le chercheur cela est considéré comme une démarche contraire à celle qu'il s'applique à mener.

28 Il demeure que l'anthropologie maritime et aquatique est une science et qu'elle ne peut se réduire à la seule démarche d'expertise. Une posture qui n'est pas toujours comprise des gestionnaires et parfois difficilement explicitée par les chercheurs. Ces situations suscitent des incompréhensions entre les acteurs et nécessitent des temps d'ajustement parfois longs. Ce que nous percevons enfin est que derrière la place à donner à 
l'anthropologie dans les enjeux sociétaux, se joue aussi la capacité de formalisation pour d'autres acteurs, des méthodes et des apports qui sont chers, mais aussi propres, à la discipline. Autre question à laquelle les chercheurs en anthropologie devraient aussi se consacrer.

Parvenir à mieux expliciter les contributions scientifiques de l'anthropologie aux autres acteurs ne pourra qu'être positif et stimulant. In fine, nous pensons que l'ensemble des questionnements relatifs à l'implication de l'anthropologie maritime vis-à-vis des préoccupations sociétales participe au renouvellement de la portée heuristique de ce champ. Au lieu d'y voir un risque d'enfermement, nous considérons que la clarification de ces positionnements sera plutôt une force.

\section{AUTEURS}

\section{NASTASSIA REYES}

Post-doctorante en anthropologie - UMR MARBEC (IRD-IFREMER-UM2-CNRS) et Chercheure associée - UMR 7206 Éco-anthropologie et Ethnobiologie (CNRS-MNHN)

Reyes.nastassia@gmail.com

\section{CLÉMENT GARINEAUD}

Chercheur associé - UMR 7206 Eco-anthropologie et ethnobiologie (CNRS-MNHN)

clement.garineaud@laposte.net

\section{FRÉDÉRIQUE CHLOUS}

Professeure d'anthropologie - Muséum national d'Histoire naturelle frederique.chlous@mnhn.fr 